\title{
Stability Measure of Compact Astrophysical Objects from Configurational Entropy Perspective
}

\author{
Nan Jiang * and Marcelo Gleiser \\ Department of Physics and Astronomy, Dartmouth College, Hanover, New Hampshire 03755, USA \\ * Author to whom correspondence should be addressed; nan.jiang.gr@dartmouth.edu.
}

Published: 5 November 2015

\begin{abstract}
We use a novel measure of shape complexity known as configurational entropy to obtain stability bounds of various astrophysical objects. We apply the method to Newtonian polytropes, neutron stars with an Oppenheimer-Volkoff equation of state, and to self-gravitating configurations of complex scalar field (boson star) in ground and excited states. The versatility of this method has also been tested on hydrogen atom in comparison with boson stars. Configurational entropic measure locates critical stability regions obtained from perturbation method with accuracy of a few percent or better.
\end{abstract}

Keywords: configurational entropy; boson star; neutron star; hydrogen atom;

\section{Introduction}

Gravitational stability of compact astrophysical objects, whether a certain equilibrium configuration is stable or not, has been a key issue in astrophysics and cosmology. To determine stability of equilibrium state of an astrophysical object one adds perturbations to the equilibrium solution of field configuration. For unstable equilibrium configurations, perturbations grows exponentially, leading to the ultimate collapse of the star. In this paper, we propose a novel method to address gravitational stability issue using a shape complexity measure called configurational entropy first brought up by Gleiser[1]. We would see that configurational entropic measure locates critical stability regions, originally obtained via perturbation method, with accuracy higher than a few percent.

Before introducing configurational entropic measure, we review essential results from application of perturbation method. One necessary but not sufficient condition of gravitational stability is that gravitational binding energy of astrophysical objects needs to be negative definitive. Gravitational 
binding energy, or the internal energy of a star[2], is defined as the energy difference between an ensemble of particles as a whole and the total energy of free particles if dispensed to infinity, i.e:

$$
E_{b}=M-m N<0,
$$

( $c=\hbar=1$ throughout) where $M$ is the total energy, $N$ is the conservative charge and $m$ is mass of the particle. It is required that $E_{b}<0$ for a stellar configuration to acquire stable equilibrium.

In this paper, we study stability of one-parameter series of stars, that is, stars obeying same physics but with different central densities $\rho(0)$ in each category (polytropes, neutrons and boson stars). An equilibrium stellar configuration with constant chemical composition and entropy per nucleon (isentropic) passes from stability to instability if only the following conditions are satisfied:[2]

$$
\begin{aligned}
& \frac{\partial E(\rho(0))}{\partial \rho(0)}=0, \\
& \frac{\partial N(\rho(0))}{\partial \rho(0)}=0 ;
\end{aligned}
$$

where $E$ is the total equilibrium energy and $N$ is the nucleon number. Readers may refer to [2] and [3] for more details.

In our cases, configurational entropy would peak at critical transition point indicated by energy or nucleon number extrema.

\section{Methods}

\subsection{Configurational Entropy}

Since we are interested in self-gravitating configurations with spatially-localized energy, consider the set of square-integrable bounded functions $f(\mathbf{x}) \in L^{2}(\mathbf{R})$ and their Fourier transforms $F(\mathbf{k})$. Plancherel's theorem states that:

$$
\int_{-\infty}^{\infty}|f(\mathbf{x})|^{2} d^{d} x=\int_{-\infty}^{\infty}|F(\mathbf{k})|^{2} d^{d} k
$$

Now define the modal fraction $f(\mathbf{k})$ [1],

$$
f(\mathbf{k})=\frac{|F(\mathbf{k})|^{2}}{\int|F(\mathbf{k})|^{2} d^{d} k},
$$

where the integration is over all $\mathbf{k}$ where $F(\mathbf{k})$ is defined and $d$ is the number of spatial dimensions. $f(\mathbf{k})$ measures the relative weight of a given mode $\mathbf{k}$. This can also be seen by noting that $|F(\mathbf{k})|^{2}$ is proportional to the Fourier transform of the two-point correlation function of the function $f(\mathbf{x})$, and $\int_{-\infty}^{\infty}|F(\mathbf{k})|^{2} d^{d} k$ is the integrated power. For periodic functions where a Fourier series is defined, 
$f(k) \rightarrow f_{n}=\left|A_{n}\right|^{2} / \sum\left|A_{n}\right|^{2}$, where $A_{n}$ is the coefficient of the $n$-th Fourier mode.

We define the configurational entropy $S_{C}[f]$ as [1]

$$
S_{C}[f]=-\sum f_{n} \ln \left(f_{n}\right)
$$

inspired by Shannon's information entropy, $S_{S}=-\sum p_{i} \log _{2} p_{i}$ [4]. Note that when all $N$ modes $k$ carry the same weight $f_{n}=1 / N$, and the discrete configurational entropy has a maximum at $S_{C}=\ln N$. If only one mode is present, $S_{C}=0$. These limits motivate the definition of Eq. 5.

For general, non-periodic functions in the continuous interval $(a, b)$, the case of interest here, the configurational entropy $S_{C}[f]$ is [1]

$$
S_{C}[f]=-\int \tilde{f}(\mathbf{k}) \ln [\tilde{f}(\mathbf{k})] d^{d} k,
$$

where $\tilde{f}(\mathbf{k})=f(\mathbf{k}) / f(\mathbf{k})_{\max }$ and $f(\mathbf{k})_{\max }$ is the maximum fraction, in many cases of interest given by the zero mode, $\mathbf{k}=0$, or by the system's longest physical mode, $\left|k_{\min }\right|=\pi / R$. This normalization guarantees that $\tilde{f}(\mathbf{k}) \leq 1$ for all physical values of $\mathbf{k}$. We call $\sigma(\mathbf{k})=-\tilde{f}(\mathbf{k}) \ln [\tilde{f}(\mathbf{k})]$ the configurational entropy density. In this paper, we will compute the configuration entropy from the energy density $\rho(r)$ of the self-gravitating object. The task at hand is thus to solve the relevant Einstein's equations to obtain the equilibrium configurations in terms of $\rho(r)$ and then use those to compute the CE as a function of the star's central density, $\rho(r=0) \equiv \rho_{0}$.

\subsection{Conventions and Fundamental Equations}

We consider static, spherically-symmetric configurations with spacetime metric,

$$
d s^{2}=B(r) d t^{2}-A(r) d r^{2}-r^{2}\left(d \theta^{2}+\sin ^{2} \theta d \phi^{2}\right),
$$

and take $c=\hbar=1$. Einstein's field equations are

$$
G_{\mu \nu}=-8 \pi G T_{\mu \nu}
$$

where $T_{\mu \nu}$ is the energy-momentum tensor. For Newtonian polytropes and neutron stars, we will model stellar matter as a perfect fluid with energy-momentum tensor

$$
T_{\mu \nu}=p(r) g_{\mu \nu}+[p(r)+\rho(r)] U_{\mu} U_{\nu}
$$

where $p(r)$ is the pressure, $\rho(r)$ is the energy density and $U^{\mu}$ is the velocity four-vector. Taking the star to be at rest, $U^{\mu}$ has only one non-zero component, $U_{0}=-\sqrt{B(r)}$. For boson stars, the energy-momentum tensor is computed from a Lagrangian density to be defined later. We use the energy density to define the mass of the object as

$$
M=4 \pi \int_{0}^{R} \rho(r) r^{2} d r,
$$


where the upper limit of the integration, $R$, is to the object's radius $R$, where $\rho(R)=0$. We would like to specifically mention that for boson stars, $\rho(r) \rightarrow 0$ at $r \rightarrow \infty$. However, we would define the effective radius within which most of the star's mass is concentrated, as:

$$
R_{\mathrm{eff}} \equiv \frac{\int_{0}^{\infty} \rho(r) r^{3} d r}{\int_{0}^{\infty} \rho(r) r^{2} d r}
$$

With these definitions, Einstein's equations can be written as:

$$
\begin{aligned}
& \frac{1}{A}\left(\frac{A^{\prime}}{A r}-\frac{1}{r^{2}}\right)+\frac{1}{r^{2}}=8 \pi G \rho ; \\
& \frac{1}{A}\left(\frac{B^{\prime}}{B r}+\frac{1}{r^{2}}\right)-\frac{1}{r^{2}}=8 \pi G p ; \\
& \frac{B^{\prime}}{B}=-\frac{2 p^{\prime}}{p+\rho},
\end{aligned}
$$

where a prime denotes derivative with respect to the radial direction. The last expression is the equation for hydrostatic equilibrium, an expression of energy-momentum conservation.

We are interested here only in isentropic stars, that is, those with a constant entropy per particle across the star. Such configurations model both very low temperature white dwarfs and neutron stars, as well as boson stars, which are self-gravitating spin-0 boson-condensates. These equations, together with an equation of state $p(r)=p[\rho(r)]$, are used to study a large variety of self-gravitating objects, assuming that $A(0)=1$ and $B(r \rightarrow \infty)=1$. The equation involving $A(r)$ and $\rho(r)$ may be integrated as $A(r)=[1-2 G \mathcal{M}(r) / r]^{-1}$, where the mass density function is given by $\mathcal{M}(r) \equiv \int_{0}^{r} 4 \pi r^{\prime 2} \rho\left(r^{\prime}\right) d r^{\prime}$.

\section{Results and Discussion}

\subsection{Polytropes}

Newtonian polytropes are obtained from the hydrostatic equation[2]:

$$
\frac{d}{d r}\left[\frac{r^{2}}{\rho(r)} \frac{d p(r)}{d r}\right]=-4 \pi G r^{2} \rho(r) .
$$

Eq. 13 is supplemented by a general polytropic equation of state

$$
p=K \rho^{\gamma},
$$

where the constant $K$ depends on the entropy per nucleon and chemical composition. $\gamma$ is the adiabatic index. Small mass, stable non-relativistic white dwarfs are well-modeled by $\gamma=5 / 3$ and $K=\frac{\hbar^{2}}{15 m_{e} \pi^{2}}\left(\frac{3 \pi^{2}}{m_{N} \mu}\right)^{5 / 3}$, where $m_{e(N)}$ is the electron (nucleon) mass, and $\mu \sim 2$ is the number of nucleons per electron. The largest mass white dwarfs are modeled by $\gamma=4 / 3$ and $K=\frac{\hbar}{12 \pi^{2}}\left(\frac{3 \pi^{2}}{m_{N} \mu}\right)^{4 / 3}$, the Chandrasekhar limit [2]. The binding energy for polytropes with $Q$ nucleons, $E_{\mathrm{bind}}=M-Q m_{N}$, can be written as $E=-\frac{(3 \gamma-4)}{(5 \gamma-6)} \frac{G M^{2}}{R}$, where $M$ is given by Eq. 10 . There is a clear stability boundary at 
$\gamma=4 / 3$ where $E$ changes sign. We will show below that the configuration entropy captures the same boundary.

Via transformation of variables, Eqs. 13 and 14 can be written as simpler Lane-Emden equation whose solution can be obtained numerically as ordinary differential equation with boundry condition $\rho(0)=\rho_{0}$ and $\rho^{\prime}(0)=0$. The CE is computed from the energy density using Eq. 6. Since polytropes have a finite radius (where $\rho(R)=0$ or, equivalently, $\theta\left(\xi_{R}\right)=0$, with $\xi_{R} \equiv R / \alpha$ ), the $k$-integration is in the interval $k \in\left[k_{\min }=\pi / R, \infty\right)$. This ensures that only modes with wavelengths smaller than the polytrope contribute to the configurational entropy.

For polytropes, there exists a simple scaling relation between the stellar mass and its configurational entropy. One can easily reach that:

$$
S \rho_{0}^{-1} \propto M^{-1} \propto \rho_{0}^{(4-3 \gamma) / 2}
$$

Note that at $\gamma=4 / 3$ the quantity $S \rho_{0}^{-1}$ is independent of $\rho_{0}$, consistent with a boundary in the star's stability [2]: stars with $\gamma<4 / 3$ are unstable, while stars with $\gamma>4 / 3$ are stable. $\gamma=4 / 3$ defines an instability ridge for the family of stellar configurations.

In Fig. 1 we show the contour plot of the stellar mass as a function of $\rho_{0}$ and $\gamma$, where the existence of a saddle ridge at $\gamma=4 / 3$ is clear. In Fig. 2 we show the contour plot of the quantity $S \rho_{0}^{-1}$ as a function of $\rho_{0}$ and $\gamma$. The reader can verify that the shadings are approximately reversed for the two plots, illustrating qualitatively the inverse scaling between mass and configurational entropy discussed above.

We have already obtained results purely analytically for Newtonian configuration. We now have the confidence to move on to general relativistic objects.

\subsection{Neutron Stars}

As we move into general-relativistic objects, we start with a simple but representative model, neutron stars with an Oppenheimer-Volkoff (OV) equation of state, where neutrons are treated as a pure ideal Fermi gas [5]. We are interested in how the effects of general relativity impacts stars' stability. We expect to locate the stability-instability transition point of OV neutron stars with various central densities using configurational entropy.

An OV neutron stars is modeled by a gas of particles with rest mass $\mu_{0}$ obeying Fermi-Dirac statistics, the related equation of state may be written in parametric form as [5]

$$
\begin{aligned}
\rho & =K(\sinh t-t) \\
p & =\frac{1}{3} K\left(\sinh t-8 \sinh \frac{1}{2} t+3 t\right)
\end{aligned}
$$


Figure 1. Contour plot for mass of polytropes as a function of the central density $\rho_{0} / \rho_{c}$ and polytropic index $\gamma$. There is an instability ridge-a saddle line-for $\gamma=4 / 3$.

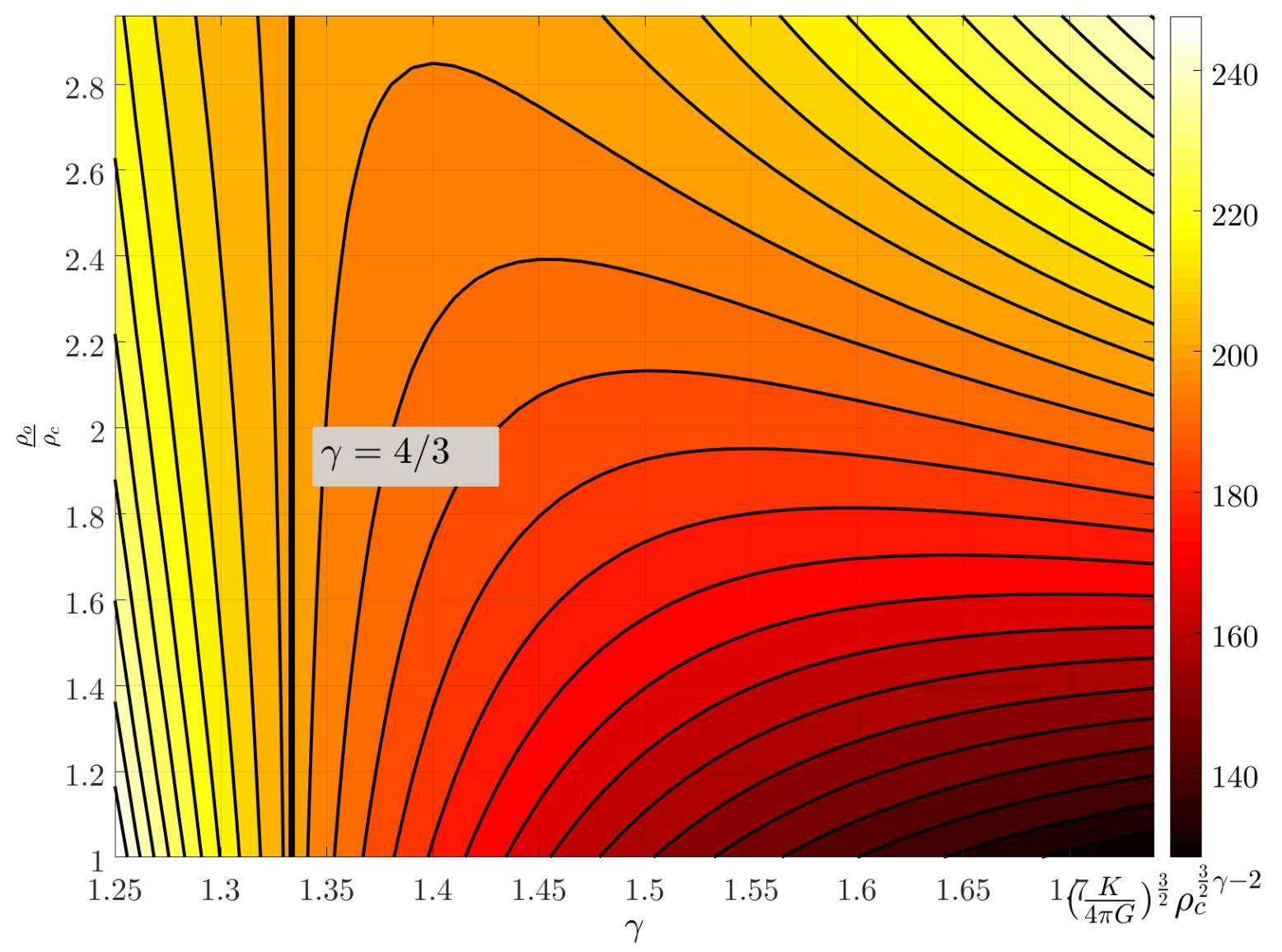


Figure 2. Contour plot for the configurational entropy of polytropes as a function of the central density $\rho_{0} / \rho_{c}$ and polytropic index $\gamma$. There is an instability ridge-a saddle line-for $\gamma=4 / 3$

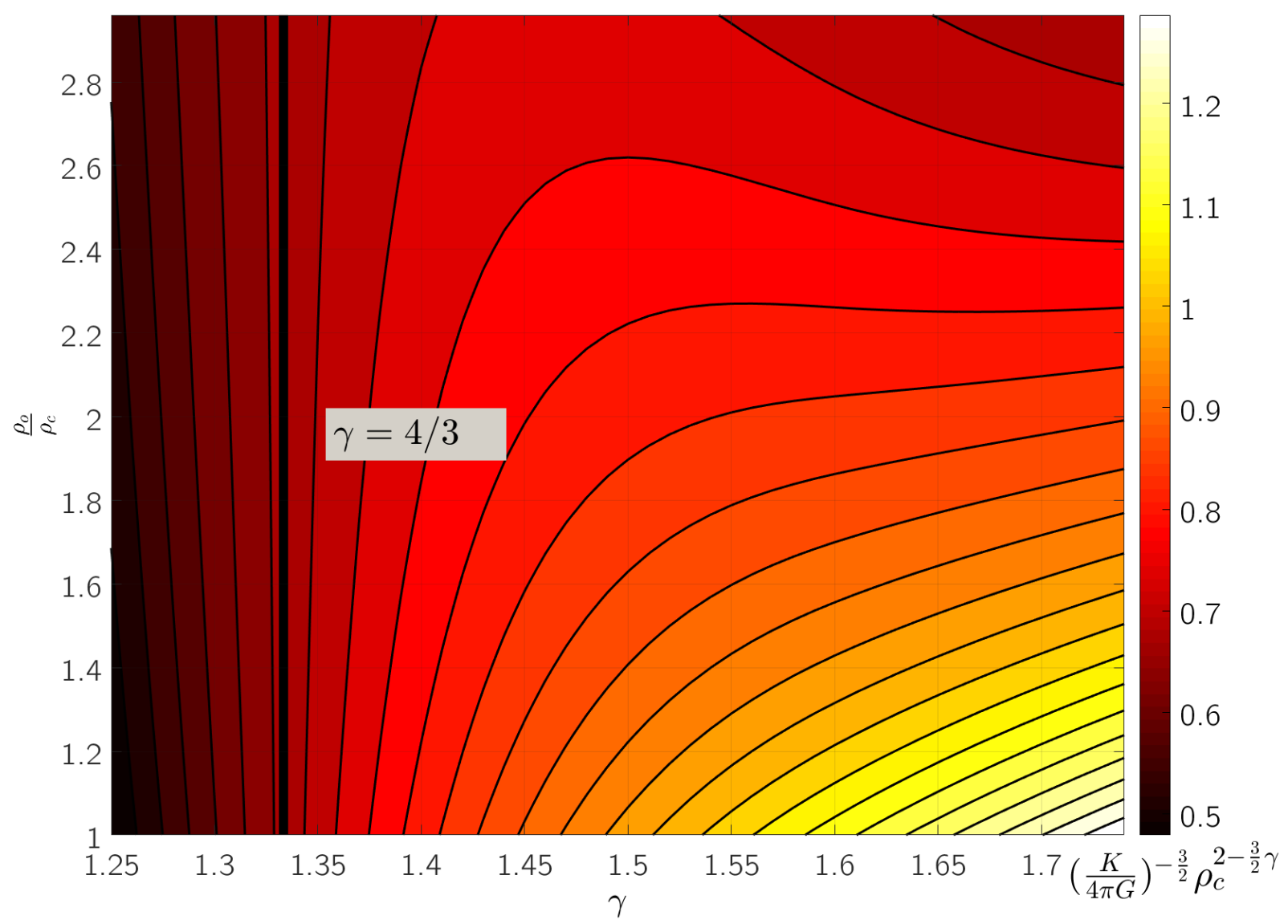


where $K=\pi \mu_{0}^{4} c^{5} / 4 h^{2}$ and

$$
t=4 \log \left(\frac{k_{F}}{\mu_{0} c}+\left[1+\left(\frac{k_{F}}{\mu_{0} c}\right)^{2}\right]^{1 / 2}\right),
$$

with $k_{F}$ being the maximum momentum in the Fermi distribution, related to the particle number density $n$ as $n=k_{F}^{3} / 3 \pi^{2} \hbar^{3}$. Readers may refer to [6] for details.

We can now solve numerically Einstein's equations with boundary conditions

$$
\begin{gathered}
u(r=0)=0 ; \quad t(r=0)=t_{0} \\
u\left(r=r_{b}\right)=u_{b} ; \quad t\left(r=r_{b}\right)=0,
\end{gathered}
$$

so that $p\left(r=r_{b}\right)=\rho\left(r=r_{b}\right)=0$ and $r_{b}$ is the radius of the star and $u_{b}$ is its mass. Results are thus parametrized in terms of $t_{0}$, related to the star's central density $\rho_{0}$ by Eq. 16. In Fig. 3 we plot the mass of the OV neutron star for values of the central density parameter $\rho_{0}$. Stars with $\rho_{0}>\rho_{c}$ are perturbatively unstable to gravitational collapse.

Figure 3. OV neutron star mass vs. central density $\rho_{0}$. Stars with $\rho_{0}>\rho_{c}=0.588$ are known to be unstable to gravitational collapse.

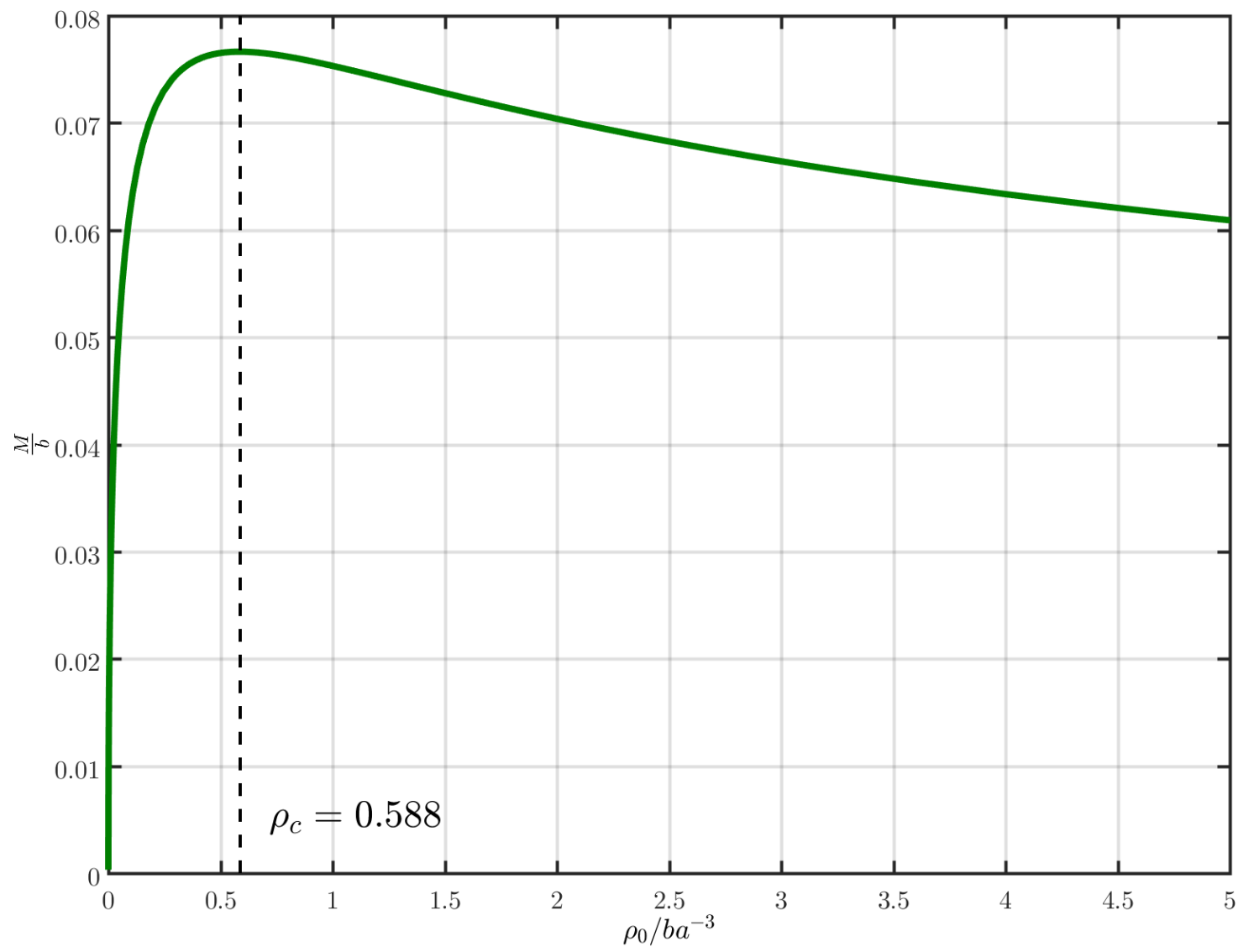

As with the Newtonian polytropes, we compute the configurational entropy using the energy density of the equilibrium configurations. The range of integration is again $k_{\min }=\pi / R \leq k<\infty$, reflecting 
the fact that neutron stars have well-defined radii where $\rho(R)=0$. The results are shown in Fig. 4, where we can see that the quantity $S \rho_{0}^{-1}$ has a minimum near (within $5.27 \%$ ) the critical equilibrium value of the central density where the stellar mass is a maximum. The inset shows the results in more detail near the CE minimum. We can translate this value of $\rho$ to an equivalent critical mass, thus establishing an upper bound in the critical OV neutron star mass with accuracy of $0.58 \%$. As with Newtonian polytropes, the configurational entropy is multiplied by the inverse central density so as to have a quantity that scales with dimensions of inverse mass.

Figure 4. Configurational entropy times $\rho_{0}^{-1}$ for the OV neutron star vs. central density $\rho_{0}$. Stars with $\rho_{0}>\rho_{c}=0.588$ are known to be unstable to gravitational collapse. The inset shows the result near the minimum.

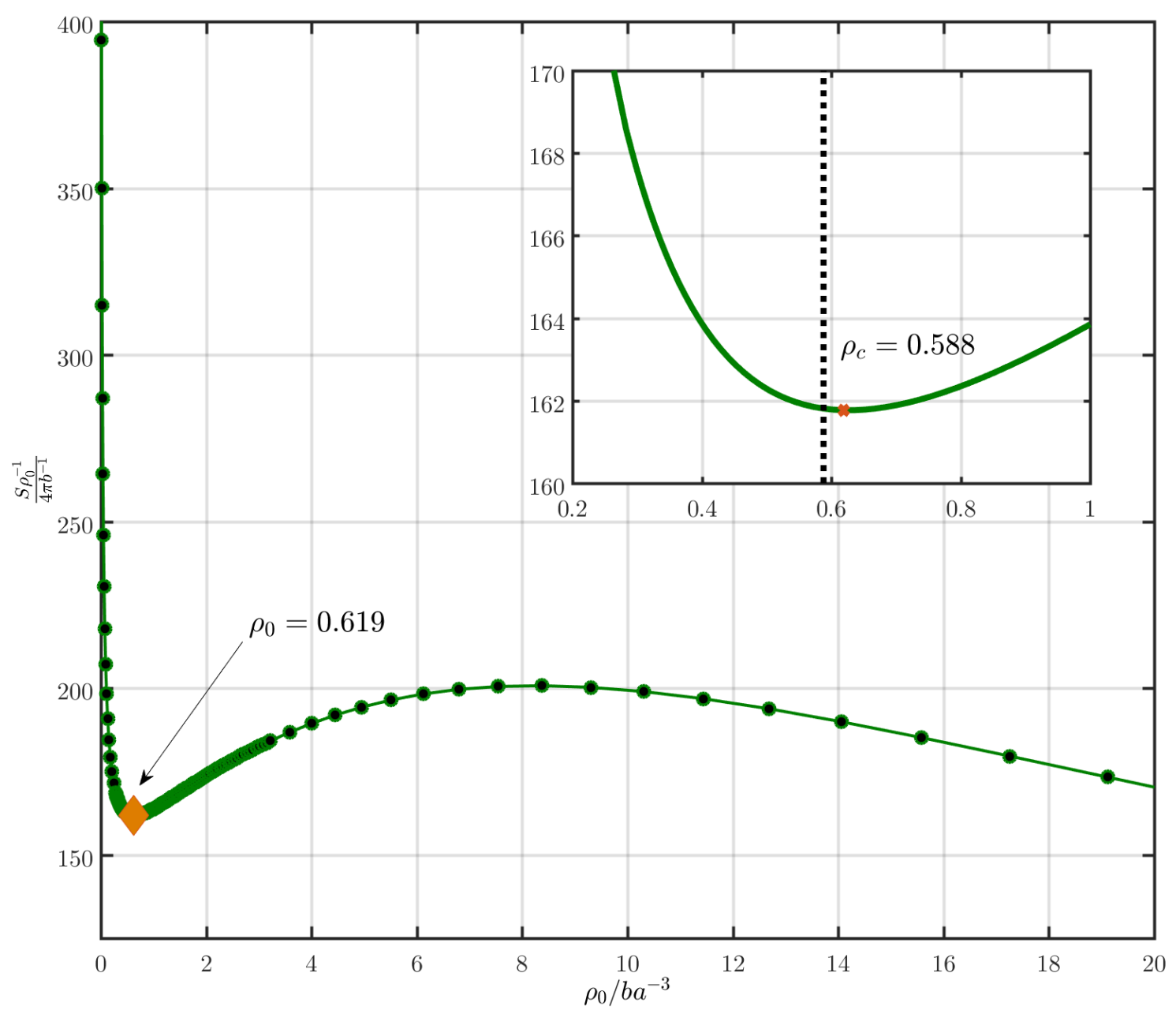

The reader should not confuse the previous results for Newtonian polytropes, where the prediction for the Chandrasekhar mass was given at the maximum of the $\mathrm{CE}$ with respect to the polytropic index $\gamma$, with the results here, where the upper-bound for the critical mass comes at the minimum of the CE with respect to central density $\rho_{0}$. The OV equation of state is only well-modeled by a polytrope in the non-relativistic limit for neutrons, with $\gamma=5 / 3$, where the star's mass is a monotonic function 
of $\rho_{0}$. The maximum mass in the mass vs. central density plot appears only when general relativistic effects are included, beyond the Newtonian approximation. We can thus see, from Fig. 4, that the configurational entropy is a reliable measure of the stability of OV neutron stars, providing an upper bound for the critical mass.

\subsection{Boson Stars}

Boson stars (BS) are hypothetical astrophysical soliton-type objects modeled by free or self-interacting massive complex scalar field carrying U(1) symmetry. While stars composed of Fermions such as white dwarfs or neutron stars are kept from gravitational collapse by Pauli's exclusion principle, boson stars are supported by pressure given by Heisenberg's uncertainty principle. Kaup[7], Ruffini and Bonazolla [8] first studied boson stars as self-gravitating particles. Relativistic treatment has found the critical mass $0.633 M_{p l}^{2} / m$ and total particle number $0.653 M_{p l}^{2} / m^{2}$ of free boson condensate (also called mini-boson star) in ground state. Colpi, Shapiro and Wasserman [9] later introduce a self-repulsive potential $\lambda|\Phi|^{4}$ which leads to star mass the order of $M_{p l}^{3} / m^{2}$ and particle number $M_{p}^{3} l / m^{3}$. The mass and particle number scales now are of the same order of magnitude of normal Fermion stars. In this section, we will briefly review the general formalism of boson stars and demonstrate how configurational-entropic method can locate boson star stability to instability transition point with an accuracy of a few percent.

\subsubsection{Formalism}

For completeness, we briefly review the essential formalism to find boson stars. Consider the action:

$$
S=\int d^{4} x \sqrt{-g}\left\{\frac{R}{16 \pi G}+\mathcal{L}\right\}
$$

and Lagrangian density,

$$
\mathcal{L}=g^{\mu \nu} \partial_{\mu} \phi \partial_{\nu} \phi^{*}-m^{2}|\phi|^{2}-\frac{\lambda}{4}|\phi|^{4} .
$$

Assuming spherical symmetry, we write the complex scalar field as $\phi(r, t)=\Phi(r) e^{-i \omega t}$, where $\Phi(r)$ is real and has $n$ nodes. For ground state boson stars, $n=0$. Stars can be found in excited states where $n \geq 2$ and their decay properties have interesting consequences, including the generation of gravitational wave bursts [10]. We define the dimensionless variables $x=m r$ and $\tilde{t}=m t$. Primes are derivatives with respect to $x$. We also absorb the dimensionless frequency $\tilde{\omega} \equiv \omega / m$ into the metric coefficient $B$, $\tilde{B}=B / \tilde{\omega}^{2}$ and define the dimensionless field $\sigma(x) \equiv \Phi(x) / \sqrt{8 \pi G}$. It proves convenient to rewrite the coupling constant $\lambda$ as [9]

$$
\Lambda=\lambda \frac{M_{\mathrm{Pl}}^{2}}{8 \pi m^{2}} .
$$


With these definitions, variation with respect to the metric of Eq. 7 and with respect to the scalar field give Einstein's equations and the Klein-Gordon equation as

$$
\begin{aligned}
A^{\prime} & =x A^{2}\left[\frac{\sigma^{\prime 2}}{A}+\left(\frac{1}{B}+1\right) \sigma^{2}+\frac{\Lambda \sigma^{4}}{4}\right]-\frac{A}{x}(A-1) \\
B^{\prime} & =x A B\left[\frac{\sigma^{\prime 2}}{A}+\left(\frac{1}{B}-1\right) \sigma^{2}-\frac{\Lambda \sigma^{4}}{4}\right]+\frac{B}{x}(A-1) \\
\sigma^{\prime \prime} & =-\left[\frac{2}{x}+\frac{1}{2}\left(\frac{B^{\prime}}{B}-\frac{A^{\prime}}{A}\right)\right] \sigma^{\prime}-A\left[\left(\frac{1}{B}-1\right) \sigma-\frac{\Lambda}{2} \sigma^{3}\right] .
\end{aligned}
$$

These equations are solved for the boundary conditions $A(0)=1 ; B(\infty)=1 ; \sigma(0)=\sigma_{0} ; \sigma(\infty)=$ $0 ; \sigma^{\prime}(0)=0$. Note that solutions are thus parametrized by the dimensionless central value of the scalar field $\sigma_{0}$, which determines the star's central density.

In Figure. 5 we plot the boson star mass and conserved charge for the free field case $(\Lambda=0)$. Note that the maximum mass is also where the binding energy $E_{b}$ is maximal, where $E_{b}=M-Q m$ (lower line). As shown in Refs. [11] the maximum mass is also the stability boundary for the boson star. This is also the case for the interacting case. Note also that stars with $\sigma_{0}>0.540$ have $E_{b}>0$, and are thus unstable to fission.

\subsubsection{Configurational Entropy of Ground State Boson Stars}

We now compute the configurational entropy for boson stars from Eq. 6 using the Fourier transform of the energy density as we did with Newtonian polytropes and neutron stars. This means that for each value of $\sigma_{0}$ we find the solution of the Einstein-Klein-Gordon system of equations and use it to compute the star's energy density $\rho(r)$. We do this for several values of the scalar self-coupling $\Lambda=0,10,50,100$. Note that since the scalar field only vanishes at spatial infinity, boson stars don't have a specific radius where the energy density and pressure vanish. We thus don't use a momentum cutoff, computing the CE for all momenta $0 \leq|\mathbf{k}| \leq \infty$. The results are shown in Figure. 6 as a function of the field's central value $\sigma_{0}$ for different values of the coupling $\Lambda$. The vertical lines denote the critical value of the field beyond which the star is unstable under radial, charge-conserving perturbations. It is apparent that these lines are very near the minima of CE for all values of $\Lambda$. We see that just as in the case with neutron stars, the configurational entropy provides a reliable upper bound on the star's stability.

\subsection{Excited Boson Stars and Hydrogen Atom}

In the previous section we see that configurational entropy of a stable ground state boson star decreases as its mass increases until the star reaches the stability-instability transition point where gravitational collapse is inevitable, as indicated by the minimum of configurational entropy. We thus expect that excited boson stars, less stable than ground state boson stars, possess smaller configurational entropy. Reference [10] predicts that a boson star in excited state may undergo spontaneous decay into ground state, generating gravitational wave bursts. 
Figure 5. Boson star mass vs. central value of the scalar field $\sigma_{0}$. Stars with $\sigma_{0}>\sigma_{c}=0.271$ are known to be unstable to gravitational collapse.

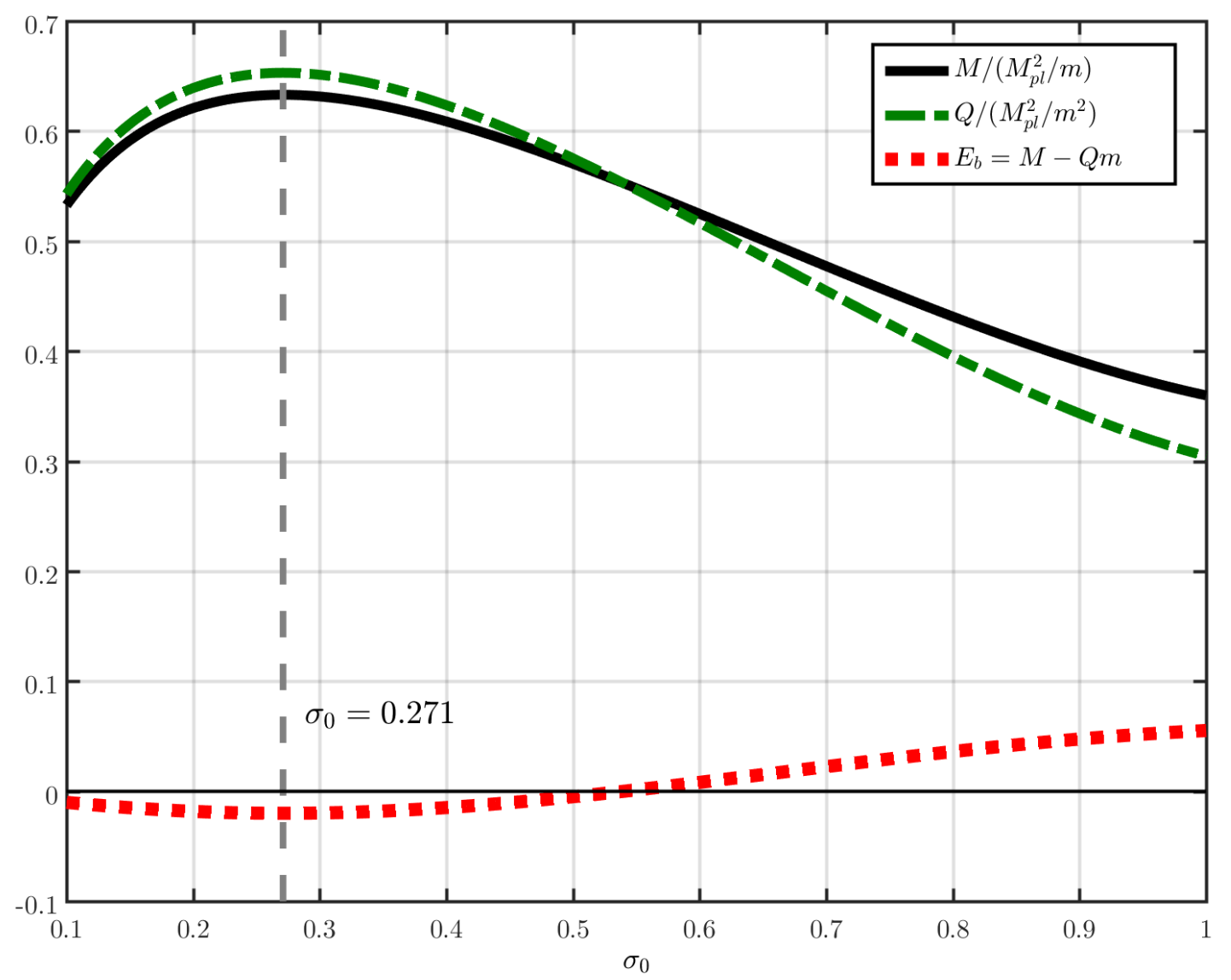


Figure 6. The configurational entropy for boson stars multiplied by inverse central density $S \rho_{0}^{-1}$ as a function of the scalar field's central value $\sigma_{0}$ for different values of the scalar field coupling $\Lambda$. The dashed vertical line denotes $\sigma_{c}$, the instability boundary for the star under radial perturbations. As in the case with neutron stars, the $\mathrm{CE}$ provides a reliable upper bound on the critical mass, with precision better than $\sim 1 \%$.
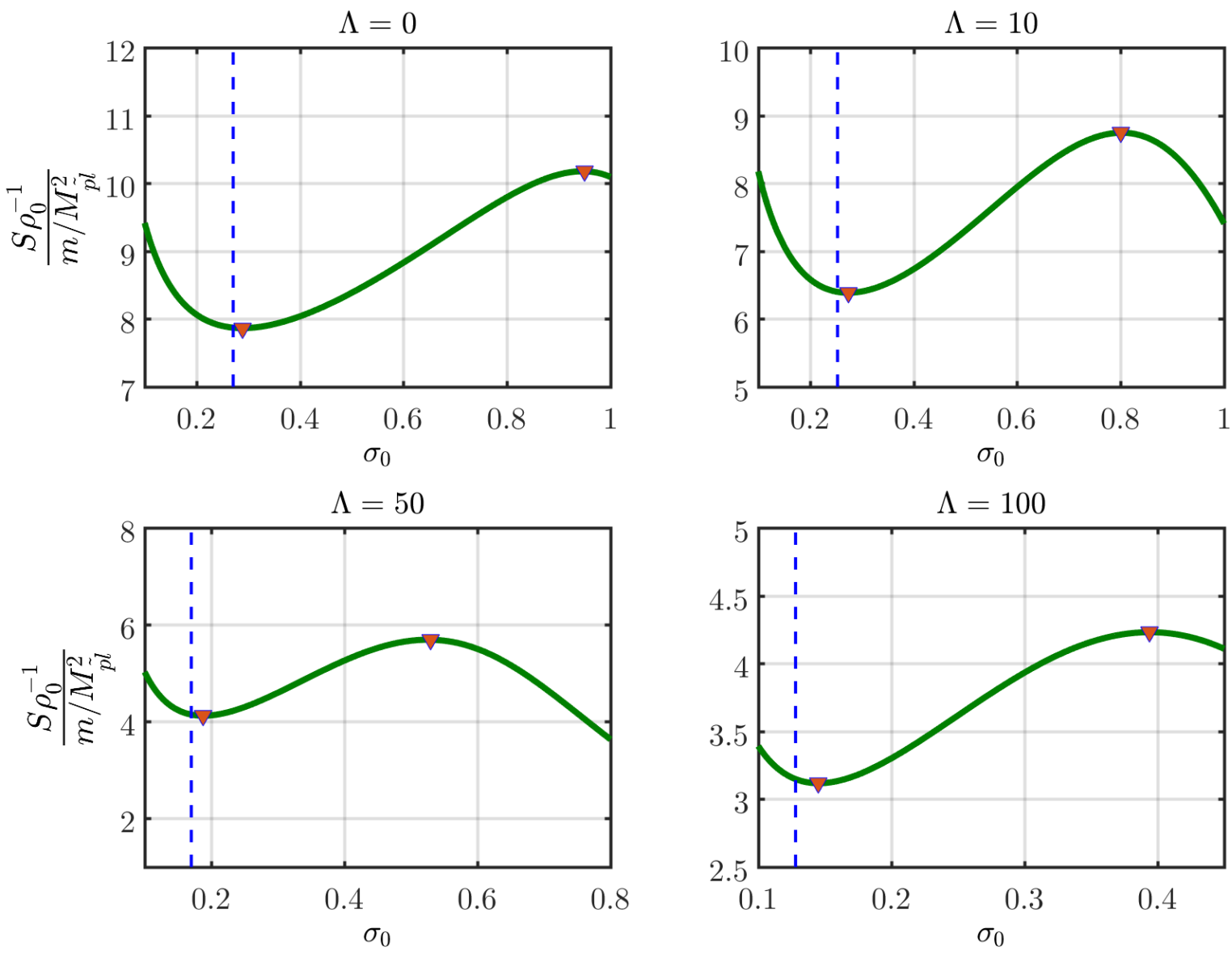
We chose four boson stars in ground, first, second and third excited state, all with the same central density $\sigma_{0}=0.4$. We then obtained their energy profiles and computed their related configurational entropy. Excited boson stars obey the same Klein-Gordon-Einstein equations, although their radial field is no longer nodeless. Field profiles for these four stars are shown in Fig. 7. Results of their configurational entropy are shown in Table.1. As we expected, the configurational entropy of a boson star decreases as it gets excited.

It is instructive to compare these results to a more familiar object. We chose to examine the configurational entropy of the hydrogen atom in its ground and excited states.

The hydrogen atom is one of the few cases where exact analytical solutions of Schroedinger's equation can be obtained. We are interested to check whether the CE could provide information about the instability of excited states. We compute numerically the configurational entropy of hydrogen using the wave function $\phi_{n l m}$. In analogy with excited boson stars, we expect that excited states $(n \geq 2)$ have smaller configurational entropy compared to ground state $(n=1)$.

Fourier transform of spherical symmetric wave function $(l=0)$ can be done in the exact same manner as in boson star. Below is a table of configurational entropy of hydrogen atom in $1 s, 2 s$ and $3 s$ states.

It can be inferred from the table that configurational entropy of hydrogen atom decreases with increasing principal quantum number, in analogy to boson stars. We conclude that configurational entropic method can be used to pick the more stable state and that the ground state of bound objects possesses the largest configurational entropy compared to other excited states.

Table 1. CE of boson stars (unit: $m^{3}$ in planck units)

\begin{tabular}{|c|c|c|c|c|}
\hline state & Ground & 1st & 2nd & 3rd \\
\hline $\mathrm{CE}$ & 4.377 & 2.444 & 1.600 & 1.163 \\
\hline
\end{tabular}

Table 2. CE of hydrogen atom (unit: $a_{0}^{-3}, a_{0}$ is Bohr radius)

\begin{tabular}{|c|c|c|c|}
\hline state & $1 \mathrm{~s}$ & $2 \mathrm{~s}$ & $3 \mathrm{~s}$ \\
\hline $\mathrm{CE}$ & 2.728 & 0.096 & 0.016 \\
\hline
\end{tabular}

\section{Conclusions}

We demostrated that for polytropes, configurational entropy has a inverse scaling relation with the mass of the star, which forms stability-instability saddle ridge at $\gamma=4 / 3$. We extend the results to fully general-relativisic neutron stars modeled with an Oppenheimer-Volkoff equation of state and to boson 
Figure 7. Field profiles for boson stars with central density $\sigma(0)=0.4$ on ground and three excited states. Ground state boson star is nodeless while other three excited stars each hit zero 1,2 or 3 times.

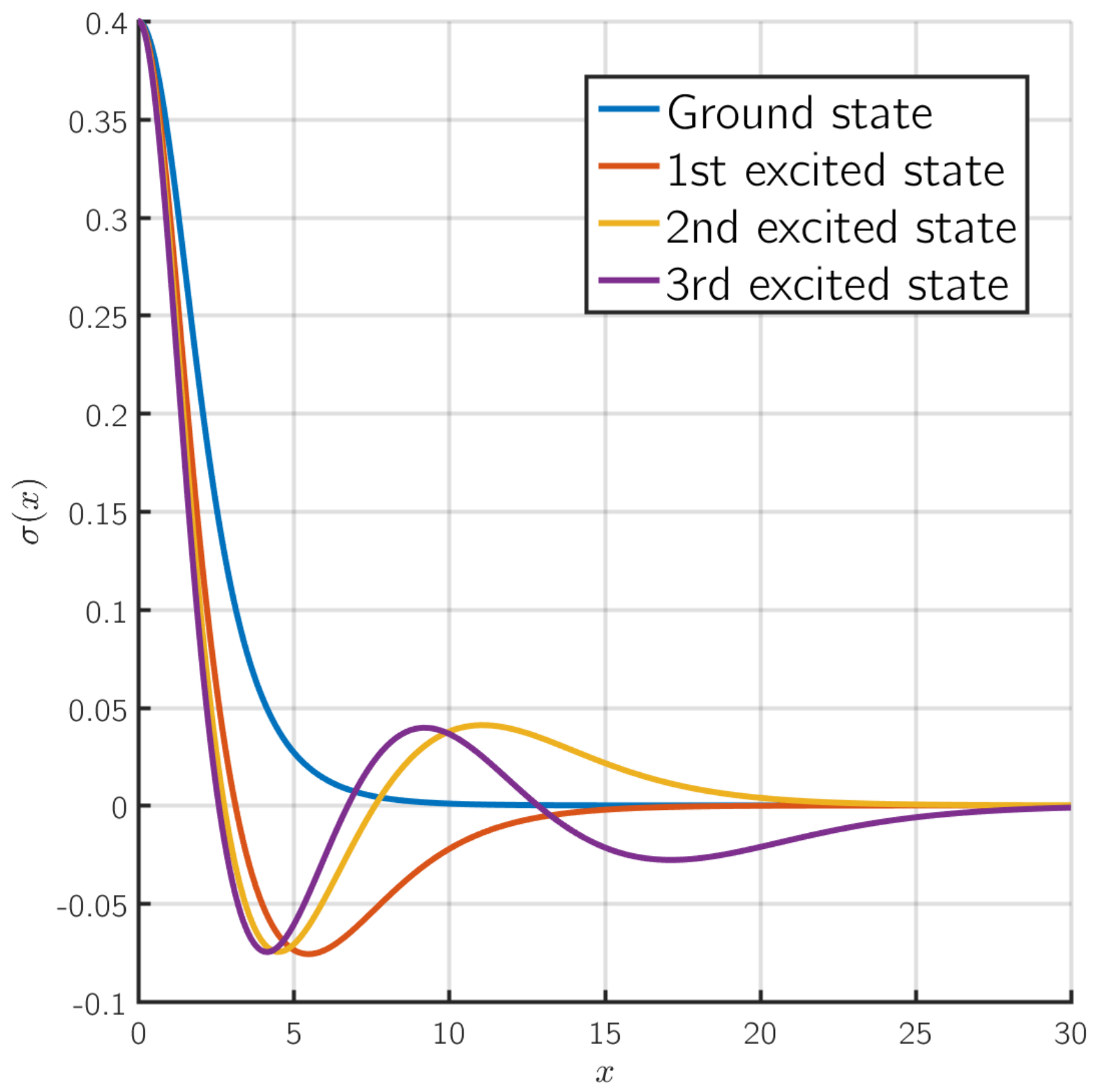


stars made of self-interacting complex scalar fields. Using the energy density of the configurations to compute their respective configurational entropy, we were able to obtain predictions to the critical stable mass with precision better than one percent for all these objects. Our approach should be seen as an alternative to usual perturbation techniques based on finding unstable eigenvalues [2,3]. Given that finding the eigenfuctions to determine perturbative stability can often be quite taxing, the configurational entropy offers another avenue to compute the critical mass of a variety of stellar objects. We also present tentative approch to compare the stability of boson stars in ground or excited states and tested using hydrogen atom model.

One question of great interest is to examine how to expand our method to include objects with lower symmetries, for example axial-symmetric bound states or even asymmetric bound states. In principle we should be able to compute the Fourier Transform for these cases and thus obtain the Configurational Entropy and determine their stability properties. Work in this topic is under investigation. Finally, we are also investigating the evolution of the configurational entropy during gravitational collapse. We expect that as the star becomes more localized its configurational entropy will increase. An important question is to determine whether the configurational entropy reaches a maximum when the event horizon forms and whether there is a relation between this hypothetical maximum value and Bekenstein's entropy based on the surface area of the black hole [12].

\section{Acknowledgements}

MG was supported in part by a Department of Energy grant DE-SC0010386. MG and NJ also acknowledge support from the John Templeton Foundation grant no. 48038.

\section{Author Contributions}

All authors contributed extensively to the work presented in this paper.

\section{Conflicts of Interest}

The authors declare no conflict of interest.

\section{References}

1. Gleiser, M.; Stamatopoulos, N. Entropic measure for localized energy configurations: Kinks, bounces, and bubbles. Physics Letters B 2012, 713, 304-307.

2. Weinberg, S. Gravitation and cosmology: principles and applications of the general theory of relativity; Wiley, 1972.

3. Shapiro, S.L.; Teukolsky, S.A. Black holes, white dwarfs and neutron stars: the physics of compact objects; John Wiley \& Sons, 2008.

4. Shannon, C.E. A mathematical theory of communication. ACM SIGMOBILE Mobile Computing and Communications Review 2001, 5, 3-55. 
5. Oppenheimer, J.R.; Volkoff, G.M. On massive neutron cores. Physical Review 1939, 55, 374.

6. Chandrasekhar, S. The highly collapsed configurations of a stellar mass (Second paper). Monthly Notices of the Royal Astronomical Society 1935, 95, 207-225.

7. Kaup, D.J. Klein-gordon geon. Physical Review 1968, 172, 1331.

8. Ruffini, R.; Bonazzola, S. Systems of self-gravitating particles in general relativity and the concept of an equation of state. Physical Review 1969, 187, 1767.

9. Colpi, M.; Shapiro, S.L.; Wasserman, I. Boson stars: Gravitational equilibria of self-interacting scalar fields. Physical review letters 1986, 57, 2485.

10. Ferrell, R.; Gleiser, M. Gravitational atoms: Gravitational radiation from excited boson stars. Physical Review D 1989, 40, 2524.

11. Gleiser, M. Stability of boson stars. Physical Review D 1988, 38, 2376.

12. Bekenstein, J.D. Black holes and entropy. Physical Review D 1973, 7, 2333.

(C) 2015 by the authors; licensee MDPI, Basel, Switzerland. This article is an open access article distributed under the terms and conditions of the Creative Commons Attribution license (http://creativecommons.org/licenses/by/3.0/). 